\title{
Codeine misuse and dependence in South Africa: Perspectives of addiction treatment providers
}

\author{
C D H Parry, ${ }^{1.2} \mathrm{PhD} ;$ E Rich, ${ }^{3} \mathrm{MA} ;$ M C van Hout,${ }^{4} \mathrm{PhD} ;$ P Deluca,${ }^{5} \mathrm{PhD}$ \\ ${ }^{1}$ Alcohol, Tobacco and Other Drug Research Unit, South African Medical Research Council, Cape Town, South Africa \\ ${ }^{2}$ Department of Psychiatry, Faculty of Medicine and Health Sciences, Stellenbosch University, Cape Town, South Africa \\ ${ }^{3}$ Alcohol, Tobacco and Other Drug Research Unit, South African Medical Research Council, Pretoria, South Africa \\ ${ }^{4}$ School of Health Sciences, Waterford Institute of Technology, Ireland \\ ${ }^{5}$ Institute of Psychiatry, Psychology \& Neuroscience, King's College London, UK
}

Corresponding author: C D H Parry (cparry@mrc.ac.za)

Background. General practitioners are referring patients with codeine-related problems to specialist treatment facilities, but little is known about the addiction treatment providers, the kinds of treatment they provide, and whether training or other interventions are needed to strengthen this sector.

Objectives. To investigate the perspectives of addiction treatment providers regarding treatment for codeine misuse or dependence. Method. Twenty addiction treatment providers linked to the South African Community Epidemiology Network on Drug Use and the South African Addiction Medicine Society were contacted telephonically and asked 20 questions.

Results. While many participants had received training in pharmacological management of individuals with opioid dependence, only two had received specific training on codeine management. Between half and two-thirds of the treatment settings they worked in provided detoxification, pharmacotherapy, psychosocial treatment and aftercare. Very few treatment settings offered long-term treatment for codeine misuse and dependence. Participants indicated that over half of their codeine patients entered treatment for intentional misuse for intoxication, and dependence resulting from excessive or long-term use. The main barriers to patients entering treatment were seen as denial of having a problem, not being ready for change, mental health problems, stigma, and affordability of treatment. Participants identified a need for further training in how to manage withdrawal and detoxification, treatment modalities including motivational interviewing, and relapse prevention.

Conclusions. Gaps in training among treatment providers need to centre on how to manage withdrawal from codeine use and detoxification, motivational interviewing and relapse prevention. Interventions are needed to address barriers to entering treatment, including user denial.

S Afr Med J 2017;107(5):451-456. DOI:10.7196/SAMJ.2017.v107i5.12242

Misuse of and dependence on codeine-containing products is on the increase globally. ${ }^{[1]}$ The problem does not only lie with over-the-counter (OTC) products where they are available, but also with inappropriate prescribing of codeine-containing medications. ${ }^{[1,2]}$ Misuse refers to 'the use of a medicine, with or without a doctor's prescription, clearly outside of accepted medical practice or guidelines, for recreational purposes or in the framework of self-medication, in greater dosages or for longer periods than were indicated, in which the risks and problems associated with use outweigh the benefits. ${ }^{[3]}$ Dependence refers to a diagnosable psychiatric condition.

Less than $45 \%$ of prescribing professionals (mainly general practitioners (GPs)) working in the UK indicated that codeine dependence could be effectively managed in general practice settings. ${ }^{[4]}$ The corresponding percentages for Ireland and South Africa (SA) were $43 \%$ and $35 \%$, respectively. ${ }^{[5]}$ As a result, individuals who are misusing or dependent on codeine are increasingly being seen in specialist substance abuse treatment centres ${ }^{[6]}$ Research in SA has indicated that there were 435 admissions to specialist substance abuse treatment centres for codeine misuse or dependence in 2014, translating to $\sim 2.5 \%$ of individuals seen in such settings. ${ }^{[7]}$ This is fairly similar to figures from the UK (2.2\%) and Ireland (1.9\%). ${ }^{[6]}$
However, these figures do not include individuals treated at public or private psychiatric treatment facilities.

\section{Objectives}

The focus of this study was to complement previous epidemiological research ${ }^{[1]}$ and research on pharmacists ${ }^{[8]}$ and $\mathrm{GP}^{[5]}$ conducted in SA by investigating the perspectives of addiction treatment providers regarding treatment for codeine misuse or dependence. Specific objectives included documenting: (i) the kinds of professionals providing treatment; (ii) the settings in which treatment is provided; (iii) the kinds of interventions delivered; ( $i v)$ the reasons patients come to treatment for codeine-related problems; $(v)$ the barriers to their accessing treatment; $(v i)$ the training needs for addiction treatment providers in terms of addressing codeine misuse and dependence; (vii) the best means for receiving additional training, should it be required; and (viii) ways to improve treatment or initiate innovations in this area. Similar research conducted in the UK and Ireland will be reported in detail elsewhere. This study was conducted as part of the SA arm of the comprehensive, multicountry Codeine Use, Misuse and Dependence (CODEMISUSED) study funded by the European Commission to inform the design of pharmacy screening and brief interventions, risk management, monitoring and surveillance, 
continuing professional development training, and the development of specific clinical/community pharmacy treatment protocols.

\section{Methods \\ Design}

The study was cross-sectional.

\section{Sampling}

Participants comprised 15 addiction treatment providers from centres participating in the South African Community Epidemiology Network on Drug Use (SACENDU) and five members of the South African Addiction Medicine Society (SAAMS). SACENDU (http:// www.mrc.ac.za/adarg/sacendu.htm) comprises 78 treatment centres across SA's nine provinces. The 15 participants were selected from 11 treatment centres with the highest number of patients in treatment for codeine-related problems in 2014. The five members of SAAMS (http://www.saams.co.za/) comprised members of the executive committee of that society. To qualify for inclusion in the study, participants had to have treated someone who had either misused codeine or was dependent on codeine in the past 12 months. This number was settled on based on expectations of the number of participants that we would need to reach before we would get saturation based on SACENDU data on the number of centres where codeine patients had received treatment in 2014, typical practice in other qualitative studies, ${ }^{\left[{ }^{[9]}\right.}$ and the human resources and funding available.

\section{Procedures}

The focus of the interview was on the providers' experience of patients/ clients as a collective, and it lasted between 20 and 30 minutes. Following invitation via the treatment listing, and receipt of informed consent, interviews were conducted telephonically by the principal investigator (CDHP) or a senior scientist (ER) who has training in research psychology, with support from seconded research staff who were given training on how to take notes during the interviews. Ethical approval for the study was required, and was granted by the South African Medical Research Council (ref. no. EC022-11/2013).

\section{Instrument}

A semi-structured interview comprising 20 questions (8 qualitative and 12 quantitative) was conducted with the addiction treatment providers. The interview drew on questions asked in the CODEMISUSED surveys of GPs and pharmacists as well as on a report on OTC and prescription-only medicines produced by the National Treatment Agency for Substance Misuse in the UK. ${ }^{[10]}$ It included sections covering demographics of respondents and treatment setting characteristics, experience with treating codeine patients, and general questions about treatment for individuals with codeine dependence, including new approaches or innovations.

\section{Data analysis}

The quantitative data were analysed using descriptive statistics (e.g. frequencies and cross-tabulations). The qualitative data that emerged from the open-ended questions in the survey were coded and analysed using thematic analysis. As the number of participants was low and the data emerging from the qualitative questions were straightforward, the themes were coded by hand.

\section{Results}

\section{Profile of participants and their treatment settings}

Three-quarters of respondents were female, with the highest proportion being in the 30 - 49-year age bracket (Table 1). The respondents held a variety of posts and worked in a number of specialties, $45 \%$ being social workers. Half of the respondents had $\leq 10$ years of service. The proportion who had received specialist training was low, with only two of 20 participants (both addiction medicine specialists) indicating that they had received specific training on codeine management, one in Australia and one via a conference. However, many had received training in the management of individuals with opioid dependence, including pharmacotherapy (methadone and buprenorphine-naloxone).

With regard to the types of treatment setting in which the respondents worked, roughly two-thirds worked in partially state-

\begin{tabular}{ll}
\multicolumn{2}{l}{ Table 1. Demographic profile of respondents $(\mathbf{N = 2 0})$} \\
\hline \multicolumn{1}{l}{$\boldsymbol{n}(\%)$} \\
\hline Gender & $5(25.0)$ \\
Male & $15(75.0)$ \\
Female & \\
Age (years) & $2(10.0)$ \\
$20-29$ & $14(70.0)$ \\
$30-49$ & $4(20.0)$ \\
$50-69$ & \\
Duration of practice in substance abuse \\
(years) \\
1 - 10 \\
$11-20$ & $10(50.0)$ \\
$\geq 21$ & $8(40.0)$ \\
Profession & $2(10.0)$ \\
Psychiatrist & \\
Psychologist & $4(20.0)$ \\
Social worker & $1(5.0)$ \\
Addiction counsellor & $9(45.0)$ \\
Nurse & $1(5.0)$ \\
Specialist methadone-prescribing doctor & $2(10.0)$ \\
BPsych or other training in psychology & $0(0)$ \\
Received any specialist training in codeine & $3(15.0)$ \\
misuse/dependence & \\
Yes & $2(10.0)$ \\
No & $18(90.0)$ \\
&
\end{tabular}

Table 2. Type of setting in which practitioners work and nature of treatment $(N=20)$

\begin{tabular}{ll} 
& $\boldsymbol{n}(\mathbf{\%})$ \\
\hline Setting & \\
Fully state-funded treatment centre & $2(10.0)$ \\
Partially state-funded treatment centre & $13(65.0)$ \\
Private practice (for profit) & $1(5.0)$ \\
Registered charity & $0(0)$ \\
Private practice with other partners & $2(10.0)$ \\
Sole private practice & $0(0)$ \\
Other - community mental health service & $0(0)$ \\
Other & $2(10.0)$ \\
Client location & \\
Urban & $14(70.0)$ \\
Rural & $2(10.0)$ \\
Mixed & $4(20.0)$ \\
Type of treatment & \\
Inpatient & $8(40.0)$ \\
Outpatient & $4(20.0)$ \\
Mixed & $8(40.0)$ \\
&
\end{tabular}


funded treatment centres (Table 2). Few of the settings catered to predominantly rural clients, with most being located in urban settings. Only four of the participants indicated that they worked in settings catering for outpatients alone. Most of the treatment carried out in SA extended over a period of $\leq 10$ weeks, and included pharmacotherapy, psychosocial therapy, cognitive behavioural therapy, motivational interviewing, psychodynamic therapy and support groups.

\section{Type of treatment provision for codeine misuse and dependence}

The number of codeine patients seen per treatment provider per year varied substantially, ranging between 1 and 250 (mean 41.4) The largest numbers of patients with codeine misuse or dependence were treated by addiction medicine specialists (on average 107.4 per year), but the data were very skewed, and the median number of codeine patients seen per year for all 20 providers interviewed was only 6 and the mode was 1 . Over half of the settings in which the practitioners worked provided detoxification, pharmacotherapy (e.g. buprenorphine-naloxone, buprenorphine or methadone) and psychosocial therapy (cognitive behavioural therapy (CBT), motivational interviewing (MI) and psychodynamic therapy), with CBT being the most common, followed by MI and aftercare (Table 3 ). Seven of the practitioners indicated that the settings in which they worked provided other treatment modalities such as support groups (including Narcotics Anonymous and Alcoholics Anonymous), family intervention therapy, lectures and alternative therapies such as reflexology, relaxation therapy, acupuncture, aromatherapy, drama therapy and occupational therapy.

Six out of 10 treatment programmes were described as fixed and not specifically tailored to patients' individual needs. Psychosocial interventions were most common, followed by detoxification and pharmacotherapy (e.g. buprenorphine). Roughly three-quarters of all treatment for codeine misuse was $\leq 10$ weeks in duration, spread fairly evenly between the $\leq 4$ weeks, 5 - 6 weeks and 7 - 10 weeks categories.

\section{Characteristics of codeine patients}

Therapeutic misuse and dependence for pain/opiate withdrawals, and intentional misuse for intoxication, were reported as main rea-

\begin{tabular}{ll}
$\begin{array}{l}\text { Table 3. Nature of treatment for codeine misuse and } \\
\text { dependence }\end{array}$ \\
\hline \\
\hline $\begin{array}{l}\text { Type of intervention } \\
\text { Detoxification }\end{array}$ \\
Pharmacotherapy \\
Psychosocial treatment & $11(55.0)$ \\
Aftercare & $11(55.0)$ \\
Other & $13(65.0)$ \\
Nature of treatment & $13(65.0)$ \\
Flexible & $7(35.0)$ \\
Fixed & \\
Both & $8(40.0)$ \\
Average length of treatment (weeks) $(N=18)$ & $12(60.0)$ \\
$\geq 25$ & $0(0)$ \\
$21-24$ & $1(5.6)$ \\
$11-20$ & $1(5.6)$ \\
$7-10$ & $2(11.1)$ \\
$5-6$ & $5(27.8)$ \\
$\leq 4$ & $4(22.2)$ \\
${ }^{*}$ Not mutually exclusive. & $5(27.8)$ \\
&
\end{tabular}

sons for seeking treatment (Table 4). Participants reported a variety of specific barriers to treatment for individuals experiencing codeine misuse and dependence. Most common was the report that patients were in denial of a problem, not ready for change, and/or had mental health problems. Barriers related to affordability (60\%), not knowing where to go for treatment, and stigma associated with going to a drug treatment centre (85\%) also rated very high.

In the event of treatment of a patient with coexisting pain and addiction to codeine medicines, participants observed difficulties in the treatment of physical pain (sometimes also emotional pain) and opiate dependence at the same time, requiring integrated pain and addiction specialist services as well as primary care involvement and specific care pathways. Participants referred to the need to provide alternative non-opioid pain relief in such patients $(n=12)$; the need to refer to outside services, and particularly for pain specialists/clinics, psychologists, psychiatrists or GPs; and the need for physiotherapy, biokinetics, acupuncture, relaxation therapy and even antidepressants.

Education and training of addiction treatment providers All the participants felt that they would benefit from further training in the area of codeine misuse and dependence (Table 5), and that this training would be most useful in the form of a face-to-face multidisciplinary workshop. When questioned around availability of best-practice guidelines for the treatment of codeine misuse and dependence, participants reported that specific protocols on the treatment and detoxification of patients who are dependent on codeine, and how best to address less severe codeine-related problems, would be very helpful. Four of the five members of SAAMS referred to their SAAMS guidelines for managing opiate dependence. Three participants from other treatment centres also referred to general opiate guidelines, which are not specific to codeine and often

Table 4. Reasons why codeine-dependent patients came for treatment, and barriers to treatment $(N=20)$

\begin{tabular}{|c|c|}
\hline & $n(\%)$ \\
\hline \multicolumn{2}{|l|}{ Reasons for treatment ${ }^{*}$} \\
\hline $\begin{array}{l}\text { Therapeutic misuse and dependence for pain and } \\
\text { opiate withdrawals (iatrogenic dependence) }\end{array}$ & $9(45.0)$ \\
\hline $\begin{array}{l}\text { Intentional misuse for intoxication and dependence } \\
\text { in response to excessive/long-term use }\end{array}$ & $13(65.0)$ \\
\hline Intentional misuse to manage anxiety & $0(0)$ \\
\hline \multicolumn{2}{|l|}{ Barriers to treatment ${ }^{*}$} \\
\hline Denial of problem & $16(80.0)$ \\
\hline Not ready for change & $10(50.0)$ \\
\hline Fear of treatment & $7(35.0)$ \\
\hline $\begin{array}{l}\text { Other mental health problems } \\
\text { (e.g. depression, anxiety) }\end{array}$ & $11(55.0)$ \\
\hline Lack of awareness of where to go for treatment & $7(35.0)$ \\
\hline Stigma associated with going to drug treatment centre & $17(85.0)$ \\
\hline Language barriers & $2(10.0)$ \\
\hline Child-care issues & $6(30.0)$ \\
\hline Geographical location of treatment centres & $2(10.0)$ \\
\hline Belief that treatment is not effective/does not work & $5(25.0)$ \\
\hline Affordability of services & $12(60.0)$ \\
\hline Transport costs and barriers & $0(0)$ \\
\hline Unaware they are addicted & $8(40.0)$ \\
\hline Others $^{\dagger}$ & $6(30.0)$ \\
\hline
\end{tabular}


Table 5. Training needs in the area of codeine misuse and dependence and best means of receiving training $(N=20)$

\begin{tabular}{ll}
\hline & $\boldsymbol{n}(\%)$ \\
\hline Training needs & \\
How to manage withdrawal/detoxification & $5(25.0)$ \\
Psychological treatment including motivational & $4(20.0)$ \\
interviewing & \\
Management of chronic pain in codeine users & $0(0)$ \\
Pharmacotherapy & $0(0)$ \\
New modalities and innovations in treatment & $3(15.0)$ \\
Early identification of codeine misuse & $0(0)$ \\
Depression and codeine misuse/dependence & $0(0)$ \\
Types of codeine use and effects & $1(5.0)$ \\
Relapse prevention & $3(15.0)$ \\
Where to make referrals & $1(5.0)$ \\
All aspects of codeine use and treatment & $1(5.0)$ \\
Best form of further training* & \\
Internet-based training & $5(25.0)$ \\
Face-to-face lecture & $6(30.0)$ \\
Face-to-face workshop & $16(80.0)$ \\
One-week course & $4(20.0)$ \\
Other (audit and practice record keeping) & $3(15.0)$ \\
${ }^{*}$ Not mutually exclusive. &
\end{tabular}

have a focus on heroin, and three treatment providers stated that they used internally developed guidelines. Ten participants indicated that they were not sure or were unaware of any such guidelines for codeine-specific cases, or stated categorically that there were no such guidelines. One participant stated that their rule of thumb was to use buprenorphine-naloxone and to stay away from benzodiazepines.

\section{Awareness of new approaches, best practices and innovations in the treatment of codeine misuse and dependence}

Twelve treatment providers were unaware of new approaches to the management of codeine misuse. Seven of the participants, however, mentioned the Codeine Care Initiative (http://selfcare247.co.za/247codeine-care-initiative/). There seems to be some doubt as to how well the Codeine Care Initiative is working. Additional comments centred on the need for patient education $(n=4)$ and the need for better training of health professionals, including psychiatrists, pharmacists, and in particular GPs $(n=9)$. Increased pharmacovigilance was also stressed by several participants, together with increasing aftercare services following treatment of individuals misusing or dependent on codeine, better sharing of information within and across sectors, and increasing community-based addiction services in general.

\section{Discussion}

The majority of addiction treatment providers who participated were female, were aged 30 - 49 years, and had 1 - 10 years' experience in substance abuse treatment provision. The most common profession was social work, followed by psychiatry. Only two of the 20 participants confirmed that they had received specialist training in codeine misuse and dependence. Over half of the settings in which the practitioners worked provided detoxification, pharmacotherapy and psychosocial therapy. In common with other studies, denial of having a problem was listed as the biggest barrier preventing individuals misusing or dependent on codeine from coming to treatment, followed by not being ready for change and lack of awareness of where to go for treatment. ${ }^{[1-13]}$ Research has shown that codeine users can feel ashamed when they realise they are dependent. Many perceive themselves as respectable, professional and highly functioning, and struggle to view themselves as an 'addict'. Desperately trying to keep their problem a secret from family members and colleagues prevents many from seeking treatment. ${ }^{[13]}$

Qualitative research recently conducted in SA and Ireland furthermore underscores the lack of awareness of risk around habitforming use of medicines containing codeine and pathways towards dependence in patients who attended treatment. ${ }^{[1,15]}$ Stigma was listed as less of a problem in SA compared with data obtained from the UK and Ireland as part of the CODEMISUSED project, ${ }^{[16]}$ but in SA and Ireland child-care issues were raised more frequently than in the UK. Six out of 10 addiction treatment providers surveyed in SA indicated that affordability of services would be a barrier to codeine patients accessing treatment, compared with only $22 \%$ in Ireland and none in the UK, where there is a National Health Insurance system. ${ }^{[16]}$ In SA, public sector drug treatment facilities are only available in the Western Cape, Gauteng, Mpumalanga, KwaZulu-Natal and Limpopo provinces. ${ }^{[17]}$ Codeine misusers in rural areas in particular face many problems in accessing treatment, as many are unemployed, they generally do not have a support system for child care, and transport costs are high. In a study conducted in Cape Town, financial and geographical factors were found to be barriers to poorer people accessing substance abuse treatment in general. ${ }^{[18]}$ Most people were reluctant to spend time travelling on costly public transport, and people were also reluctant to pay for treatment, as they were often aware that there were government-funded centres available - albeit with a lengthy waiting list. ${ }^{[14]}$ Surprisingly, only $10 \%$ of our SA participants indicated that the geographical location of treatment settings would be a problem, compared with $22 \%$ for Ireland, and none mentioned transport-related costs as being a barrier for codeine patients in SA, compared with $30 \%$ for Ireland. ${ }^{[16]}$

Practitioners were optimistic about preventing relapse by the use of alternative non-opioid pain relief in patients with coexisting pain and addiction to codeine medicines. In such instances they also reported referring to other professionals such as pain specialists/clinics, psychologists and psychiatrists, and recommending alternative treatments such as acupuncture or relaxation therapy. In contrast, treatment providers in both the UK and Ireland were pessimistic about positive outcomes when dealing with patients with coexisting pain and codeine dependence, with Irish practitioners reporting that poorer outcomes were likely. ${ }^{[16]}$

Almost half of the participants highlighted the need for better training of health professionals in managing codeine-related problems. Previous research has indicated that such training is needed for a broad range of health professionals, including psychiatrists, pharmacists, and in particular GPs. ${ }^{[4]}$ Best-practice guidelines for the management of opioid use disorders have been developed by SAAMS, ${ }^{[19]}$ but few of the non-medical treatment providers surveyed were aware of them. Furthermore, these guidelines and those from countries such as Australia and New Zealand are not specific to individuals dependent on codeine and tend to focus on stronger opioids such as heroin. ${ }^{[20,21]}$ According to Nielsen et al. ${ }^{[22]}$ there are many differences between individuals seeking treatment who are dependent on codeine as opposed to strong prescription opioids. The most common reason for use of prescription opioid analgesics in both groups was to resolve pain, although only the codeine-dependent group reported initiation due to acute pain such as toothache or headaches. This could imply that the latter group have less contact with health professionals and are more likely to self-medicate. They were also less likely to report heroin use and had a higher proportion of female users. However, the main difference between the two groups 
was that the codeine group was less likely to enter maintenance treatment, which may indicate that health professionals wrongly assume that a shorter duration of treatment is needed, as they believe such patients have a milder opioid dependence. ${ }^{[22]}$ Similar to their counterparts in the UK and Ireland, ${ }^{[16]}$ our study participants believed that this training should take place in a workshop or lecture format, i.e. face to face rather than online. In addition to training in the management/treatment of codeine misuse and managing withdrawal and detoxification, SA participants (in contrast to colleagues in the UK and Ireland ${ }^{[16]}$ ) also wanted training in relapse prevention.

Several participants stressed that increased pharmacovigilance was also needed, together with increased aftercare services following treatment of individuals misusing or dependent on codeine, better sharing of information within and across sectors, and more community-based addiction services in general. ${ }^{[23]}$ Many of these interventions were also identified as being important in interviews conducted with key national stakeholders from a variety of sectors beyond addiction practice in the UK, Ireland and SA in another component of the CODEMISUSED project. ${ }^{[24]}$

\section{Study limitations}

This study is subject to several limitations. First, the number of participants was small and participants were identified by purposeful sampling, which could limit the generalisability of the findings more broadly. However, in terms of participants recruited from centres participating in SACENDU, they do represent treatment providers working in centres seeing substantial numbers of patients who are treated for codeine-related problems. The participants recruited via SAAMS $(n=5)$ may also have been biased towards those who are more senior and experienced, given that they were exco members of this society. Nevertheless, given their experience, their insights are likely to be of value. The profiles of the participants varied widely, especially with regard to professions, skills, experience and training in the field of addiction, which is likely to have had an effect on the results. More nuanced research is needed in future, to go into more depth on interventions likely to be provided by addiction treatment providers with different professional backgrounds.

\section{Recommendations}

While we do not have good outcomes data on the effectiveness of the treatment of individuals misusing or dependent on codeine in SA, we do know that over a third (37\%) of codeine patients treated in centres participating in SACENDU had previously received treatment for the same condition, and that many of the addiction treatment providers participating in the research highlighted the need for better training of health professionals in managing codeine-related problems. In view of this, specific best-practice guidelines for managing codeine misuse and dependence should be developed and/or updated to incorporate innovations to ensure that individuals misusing or dependent on codeine receive a uniform and proven treatment option. ${ }^{[14,15,25]}$ Availability of opioid agonists should probably be expanded to low-threshold services in the treatment of codeine dependence. ${ }^{[26]}$ The training needs of treatment providers should be addressed as a matter of urgency to cover best practices for managing codeine misuse and dependence, including detoxification modalities, pharmacotherapy maintenance treatment, psychosocial support and ensuring proper aftercare. ${ }^{[24]}$

Given the relatively low numbers of individuals in treatment for codeine-related problems ${ }^{[5]}$ and the possible barriers that may make it harder for them to access treatment, active steps are needed, in particular to address denial of the problem and fear of being stigmatised. This first needs to be verified by research conducted among codeine-dependent patients themselves. Among other things, appropriate responses might include increasing media coverage of the problem of codeine misuse and dependence, and public health campaigns highlighting that anyone can become addicted to codeine products when used in the long term or excessively. ${ }^{[13,24]}$ Awareness campaigns should also educate the public on where they can go to seek help.

\section{Conclusions}

There appears to be a broad spread of individuals providing treatment for persons misusing or dependent on codeine. They have general training in the provision and management of opioid agonist treatment, but few have specifically been trained in the management of codeinerelated misuse/dependence. Further training may need to centre on how to manage withdrawal and detoxification, titration of the correct dosage of agonist therapies, psychological interventions and relapse prevention. The main barriers to patients entering treatment were seen as denial of having a problem, not being ready for change, mental health problems, stigma associated with going to a drug treatment centre, not knowing where to access treatment, and affordability. While further investigation is still needed, including research among codeine patients in treatment, consideration should be given to campaigns that highlight that anyone can become dependent on codeine products used in the long term or excessively, addressing concerns associated with the stigma of going to a drug treatment centre as a person misusing or dependent on codeine, and addressing the affordability of drug treatment in general and where to go for help.

Acknowledgements. The authors were on secondment as part of the multicountry CODEMISUSED project and working with the Local Choice pharmacy group in SA (CDHP, MCvH, PD), Weldricks Pharmacy in the UK (CDHP) and Cara Pharmacy in Ireland (CDHP, PD, MCvH). We acknowledge pharmacist assistants from Ireland (Jillian Glynn and Daniel Mcgill) and a pharmacist from the UK (Mariam Deldar), who assisted us in conducting and transcribing interviews and collating participants' responses while on secondment to the SA Medical Research Council during 2015. We also thank the study participants for their willingness to be interviewed.

Author contributions. All the authors were involved with the design of the study. CDHP and ER were involved with the data collection, CDHP wrote the first draft of the manuscript with the assistance of ER, and MCvH and PD commented on subsequent drafts.

Funding. Funding was received from the European Commission's Seventh Framework Programme FP7/2007-2013 under grant agreement no. 611736. Conflicts of interest. None.

1. Van Hout MC, Bergin M, Foley M, et al. A Scoping Review of Codeine Use, Misuse and Dependence Final Report. Brussels: CODEMISUSED Project European Commission 7th Framework Programme, 2014 2. Nielsen S, van Hout MC. Over-the-counter codeine - from therapeutic use to dependence, and the grey areas in between. In: Ellenbroek B, Geyer M, Marsden C, eds. Current Topics in Behavioral
Neurosciences. Heidelberg Germany: Springer 2015:1-17.

3. Casati A, Sedefov R, Pfeiffer-Gerschel T. Misuse of medicines in the European Union: A systematic Casati A, Sedefov R, Pfeiffer-Gerschel T. Misuse of medicines in the European Union: A system
review of the literature. Eur Addict Res 2012;18(5):228-245. http://dx.doi.org/10.1159/000337028 review of the literature. Eur Addict Res 2012;18(5):228-245. http://dx.doi.org/ $10.1159 / 000337028$
4. Foley M, Carney T, Rich E, Parry C, van Hout MC, Deluca P. Medical professionals perspectives on prescribed and over-the-counter medicines containing codeine: A cross-sectional study. BMJ Open 2016;6(7). http://dx.doi.org/10.1136/bmjopen-2016-011725

5. Foley M, Carney T, Rich E, van Hout MC. Work Package 3. Survey of Medical Professionals. Brussels: CODEMISUSED Project European Commission 7th Framework Programme, 2015.

6. Parry $\mathrm{CDH}$, Deluca P, Cooper R, van Hout MC. Do we have sufficient information to optimally inform regulatory or other policy decisions about medications containing codeine? Addiction 2015;110(10):1690-1691. http://dx.doi.org/10.1111/add.13047

7. Dada S, Harker Burnhams N, van Hout MC, Parry CDH. Codeine misuse and dependence in South Africa - learning from substance abuse treatment admissions. S Afr Med J 2015;105(9):776-779. http:// dx.doi.org/10.7196/SAMJnew.8172

8. Carney T, Wells J, Bergin M, et al. A comparative exploration of community pharmacists' view on the nature and management of over-the-counter (OTC) and prescription codeine misuse in three regulatory regimes: Ireland, South Africa and the United Kingdom. Int J Ment Health Addiction 2016;14(4):351-369. http://dx.doi.org/10.1007/s11469-016-9640-z 
9. Crouch M, McKenzie H. The logic of small samples in interview-based qualitative research. Soci Sci Inf 2006;45(4):18. http://dx.doi.org/10.1177/0539018406069584

10. National Treatment Agency for the Misuse of Substances. Addiction to Medicine. London: National Health Service, 2011 .

11. Roussin A, Bouyssi A, Pouche L, Pourcel L, Lapeyre-Mestre M. Misuse and dependence on nonprescription codeine analgesics or sedative hl antihistamines by adults: A cross-sectional investigation
poussion prescription codeine analgesics or sedative h1 antihistamines by adults: A cross-sectional i
in France. PLOS One 2013;8(10):e76499. http://dx.doi.org/0.1371/journal.pone.0076499

12. Roberts S. Codeine upscheduling threatens bottom lines. Pharmacy News, 26 March 2010. http://www. pharmacynews.com.au/article/codeine-upscheduling-threatens-bottomlines/514485.aspx (accessed 28 November 2016)

13. Cooper RJ. 'I can't be an addict. I am?' Over-the-counter medicine abuse: A qualitative study. BMJ Open 2013;3:e002913. http://dx.doi.org/10.1136/bmjopen-2013-002913

14. Van Hout MC, Horan A, Santlal K, Rich E, Bergin M. 'Codeine is my companion': Misuse and dependence on codeine containing medicines in Ireland. Ir J Psychol Med 2015;1-14. http://dx.doi. org/10.1017/ipm.2015.60

15. Van Hout MC, Rich E, Dada S, Bergin M. 'Codeine is my helper': Misuse of and dependence on codeine-containing medicines in South Africa. Qual Health Res 2017;27(3):341-350. https:// www.researchgate.net/profile/Michael_Bergin2/publication/312336223_Codeine_Is_My Helper_Misuse_of_and_Dependence_on_Codeine-Containing_Medicines_in_South_Africal links/587b7dle08ae9a860fe9771d.pdf (accessed 29 March 2017).

16. Deluca P, van Hout MC, Gooney M, et al. Work Package 5. Addiction Treatment Providers' Experiences of Codeine Use, Misuse and Dependence: Final Report. Brussels: CODEMISUSED Project European Commission 7th Framework Programme, 2015.

17. Foley M, Rich E, Norman I, van Hout MC. Codeine Consumption Through Prescribing in the Republic of Ireland, the Republic of South Africa and the United Kingdom. Supplement to the CODEMISUSED of Ireland, the Republic of South Africa and the United Kingdom. Supplement to the CODEMISUSED
Scoping Review. Brussels: CODEMISUSED Project European Commission 7th Framework Scoping Review.
Programme, 2014.

Programme, 2014.
18. Myers BJ, Louw J, Pasche S. Inequitable access to substance abuse treatment services in Cape Town, South Africa. Subst Abuse Treat Prev Policy 2010;5:28. http://dx.doi.org/10.1186/1747-597X-5-28
19. South African Addiction Medicine Society. South African Guidelines for the Management of Opioid Use Disorders. Pretoria: SAAMS, 2015

20. Gowing L, Ali R, Dunlop A, Farrell M, Lintzeris N. National Guidelines for Medication-Assisted Treatment of Opioid Dependence. 2014. http://www.nationaldrugstrategy.gov.au/internet/drugstrategy/ Publishing.nff/content/AD14DA97D8EE00E8CA257CD1001E0E5D/\$File/National_Guidelines_2014. pdf (accessed 28 November 2016)

21. Ministry of Health, New Zealand. New Zealand Practice Guidelines for Opioid Substitution Treatment. Wellington: Ministry of Health, 2014. http://www.health.govt.nz/publication/new-zealand-practiceguidelines-opioid-substitution-treatment-2014 (accessed 28 November 2016).

22. Nielsen S, Murnion B, Dunlop A, et al. Comparing treatment-seeking codeine users and strong opioid users: Findings from a novel case series. Drug Alcohol Rev 2015;34(3):304-311. http://dx.doi. org/10.1111/dar.12224

23. Van Hout MC. Doctor shopping and pharmacy hopping: Practice innovations relating to codeine. Drugs Alcohol Today 2014;14(4):219-234. http://dx.doi.org/10.1108/DAT-03-2014-0014

24. Norman I, Bergin M, Parry C, van Hout MC. Best practices and innovations for managing codeine misuse and dependence. J Pharm Pharm Sci 2016;19(3):367-381. http://dx.doi.org/10.18433/J3T89K

25. Bergin M, Norman I, Foley M, et al. Practice implications and recommendations for managing codeine misuse and dependence. Acta Pharmacol 2015;65(4):351-364. http://dx.doi.org/10.1515/acph-20150040

26. Van Hout MC, Hearne E, Bergin M. Buprenorphine-naloxone in the treatment of codeine dependence: A review of cases. Int J Addict Ment Health 2017;15(1):224-237. http://dx.doi.org/10.1007/s11469016-9655-5

Accepted 18 January 2017. 